\title{
An Improvement of Leach Protocol using Cross Layer Leach - Heed and Super Cluster Head
}

\author{
Sandeep Kaur \\ M.Tech scholar \\ Department of Computer \\ Science and Engineering, \\ Shaheed Bhagat Singh State \\ Technical Campus, Ferozepur, \\ Punjab (152004), India
}

\author{
Daljeet Kaur \\ Associate Professor \\ Department of Computer \\ Science and Engineering, \\ Shaheed Bhagat Singh State \\ Technical Campus, Ferozepur, \\ Punjab (152004), India
}

\author{
Gagandeep \\ Assistant Professor \\ Department of Computer \\ Science and Engineering, \\ Shaheed Bhagat Singh State \\ Technical Campus, Ferozepur, \\ Punjab (152004), India
}

\begin{abstract}
The Wireless Sensor Networks (WSN) resides of a large number of sensor nodes that are incomplete in energy, processing power, and storage. The energy of nodes is the most important consideration amid them because the lifetime of Wireless Sensor Networks is incomplete by the energy of the nodes. LEACH is one of the most famous clustering mechanisms; it elects a cluster head $(\mathrm{CH})$ created on a probability model. This paper improves LEACH protocol using Cross Layer LEACH and HEED and Super Cluster Head. Hybrid Energy Efficient Distributed protocol for the heterogeneous wireless sensor network have been reported. The main requirements of the wireless sensor network are to prolong the network lifetime and energy efficiency. Here, Heterogeneous- HEED: A Protocol for clustered heterogeneous for Wireless Sensor Network has been proposed to prolong the network lifetime. These proposed works provide a better result in terms of o live nodes, energy dissipation, message cost when compared to traditional leach protocol.
\end{abstract}

\section{Keywords}

HEED, WSN, SCH, Protocol, Algorithm etc.

\section{INTRODUCTION}

Typical sensor nodes are able to carry out sensing, data processing and communicating components, making them feasible for a wide range of promising applications, such as environmental monitoring (e.g., humidity, temperature), disaster, healthcare, military, etc. [1]. Sensor nodes that are used for these applications are mostly deployed densely and operated independently. Sensor nodes are normally battery powered and left alone in environments, making it quite challenging to replace or recharge node batteries. Hence, one of the critical challenges in WSNs is to prolong network lifetime due to limitation of energy resources. The criticallylocated sensors are those located near the sink, which carries the burden of relaying large amounts of data traffic, especially when multiple high-rate routes pass through these nodes. Thus, avoiding the failure of such nodes caused by early energy depletion is crucial for prolong the network lifetime. Another important challenge occurs when each and every node wants to simultaneously transmit and receive data at the same time [2]. This will lead to a lot of data collisions and congestion. As a result, a large amount of energy is wasted, and most of the nodes will run out of energy very quickly. Many proposals have concentrated on improving the energy efficiency. The research in data-centric WSNs is concentrated on clustering by reducing the number of transmissions to the sink, by selection of a proper MAC layer and an energyefficient data aggregation mechanism to alleviate the challenges of WSNs. Clustering means grouping the nodes based on geographical location into different clusters. The clustering technique decreases the number of nodes required to forward data to the sink node, thereby reducing the channel load and improving the scalability [3].

However, the advantages of clustering algorithms come with additional overheads during $\mathrm{CH}$ selection and the cluster formation process. Improvement in the MAC layer can increase the reliability and increase the sleep time of nodes. The MAC layer can be based on contention, contention-free or hybrid protocols.

\section{CROSS-LAYER PROTOCOL DESIGN}

Most of the communication protocols for WSNs follow the traditional layered protocol architecture. While these protocols may achieve very high performance in terms of the metrics related to each of these individual layers, they are not jointly optimized to maximize the overall network performance while minimizing the energy consumption [4]. Considering the energy constraint and processing resources of WSNs, joint optimization, and design of networking layers, (i.e. cross-layer design), stands as the most optimistic alternative to improve trsaditional layered protocol architectures. The central idea of cross-layer design is to optimize the control and exchange of information over two or more layers to achieve significant performance improvements by exploiting the interactions between various protocol layers. An important question in the area of cross-layer design is what parameters need to be shared among different layers of the protocol stack and how can each layer be made robust to the changing network conditions. The benefits and advantages from relaxing the rigid layered structure need to be quantified, and the associated complexity and stability issues with implementing such cross-layer design need to be studied more thoroughly. The cross-layer design focuses on the interaction or modularity among physical, MAC and routing layers. Some examples of cross-layer approaches are illustrated, to introduce the SERAN protocol [5]. A estimating residual energy a efficient method is used, by consider the performance characteristics of battery according to the load and temperature in batteries, along with its voltage. This can be possible by utilizing a small database which contains the useful residual energy information measured in the various temperature and load environments.

\section{RELEATED WORK}

Wireless sensor nodes have certain constraints associated with 
them particularly minimizing energy consumption is the basic and key requirement in formulating the sensor network protocols. Since the nodes are small, it is unattendable, batteries may drain out very fast, so it is necessary that the network be energy efficient in order to maximize the lifetime of the networks [6]. It demands fault tolerance scalability, production costs and reliability[6].By using direct diffusion(DD), a query based technique for routing the packet and by aggregating, caching and reinforcement the suitable link is dynamically selected for transmission. In LEACH protocol the selection of cluster head is elected dynamically in rotation. Data fusion is adopted to reduce the amount of data send over the link in the network [3]. In continuation the time interval is partitioned into fixed intervals with equal length and updating is made for selection of $\mathrm{CH}$ here[4,5]. Sensors sense and gathers the data's of its sensing and through the data forwarding transmission technique the data's are transmitted[5,6]. Some stochastic mode of technique is adopted for cluster head selection. In HCR method each cluster is managed with the set of associates and the lifetime of the clusters last long [7]. In S-MAC concept, some random nodes are put to sleep mode for a particular time till then the traffic is controlled by storing the packet in the neighbouring nodes. When the node is $\mathrm{ON}$ it starts retrieving the packets from its neighbouring nodes. This mode selection is made in random so that the consumption of energy by the network is maintained low[8]. In some papers it is discussed that the cluster head is selected based on the number of times the node has been a cluster head so far or by choosing the random number and if its below the optimized threshold that particular nodes is given a chance as a cluster head in the network[9]. Some cases by deducing the hierarchical clustering strategy it has been found that the energy savings increases with the number of levels in the hierarchy [10]. As a conclusion, CHs are elected based on the various techniques seen above and these strategies supports the concept of reducing the residual energy in the network. Even the concept of iteration plays a vital role in electing the heads as term basis and performs the task. Critical data's are sending out to the destination immediately after sensing the status of it.

\section{POWER-EFFICIENT CLUS TERING SCHEME}

A "super" cluster that contains all sensors of the WSN would be attractive. However it is not feasible as the size of WSN grows larger. Considerations such as energy limitation, cost, and scalability make a single-cantered WSN not only unfavourable, but also difficult to implement. The proposal of hierarchical organization of WSN is to distribute the computational and managerial tasks to a group of cluster heads. This approach will reduce the communication traffic in network, and will allow the deployment of less powerful, lower priced processors to do the WSN's computational and managerial jobs in a collaborative manner. We assume each transmission round will collect data from one sensor. That is a simplification of real situations where multi-channel transmission/reception maybe supported [11].

\section{HEED ALGORITHM}

HEED clustering algorithm is a distributive clustering method that considers energy and communication value at the similar time. HEED is iterative grouping algorithm formula that utilizes the residual energy of nodes and their communication value to settle on the most effective set of cluster head nodes. Throughout clustering procedure, a sensor node may well be tentative cluster head, final cluster head or coated. At the start of grouping part, a node with higher energy state is additionally probable to be selected as a tentative cluster head. It sends a message to all or any sensor nodes existing in its cluster vary and informs them concerning its new condition[12]. For this purpose, AMRP (average power) is introduce cost criterion and is exploited for breaking constraints in the procedure of cluster head selection. This formula has four main goals[13]:

- Increasing network lifetime by distributing energy utilization

- Terminating clustering method by constant variety of iterations

- Minimizing management overload

- Providing acceptable distributed cluster heads and compressed clusters. HEED doesn't think about any assumptions concerning distribution, density or ability of nodes (such as nodes ability relative to their location). Since the formula aims to prolong network period, it decides supported the residual energy of every node. Thus, cluster vary is decided by transmitted power level utilized in inhume cluster communication and through clustering [14].

When many nodes are the candidate for being cluster head, the node that has all-time lowest inter-cluster value is going to be elite as the cluster head. This cost is a function of:

- Cluster characteristics, like the size of a cluster. [15]

\section{a)Distance calculation}

To avoid the selection of the node with low residual energy as a cluster head, the residual energy takes into consideration when the value of the threshold is calculated. Initially, the network all the sensor nodes in the network is divided into smaller groups known as clusters. These clusters further involve in cluster formation and cluster head selection. During the cluster head selection process, all the nodes in a particular cluster select a head node that is known as cluster head. The selection of cluster head depends upon on the distance from the base station and residual energy

\section{Algorithm for Cluster Head Selection Input: N, IE}

Output: Cluster Head

Begin

For all nodes N, update location;

Split node based on the location;

Compute energy for all nodes $\mathrm{N}$;

If $\operatorname{deg}($ vi) $>\mathrm{N}(\mathrm{vi})$

Select as a Node as a $\mathrm{CH}$ and allocate slot;

End If;

If $(\mathrm{N}$ IE $<\mathrm{NE} \| \mathrm{N}$ IE $=\mathrm{NE})$

If (NIDID<BD)

Select Node as Super Cluster Head and calculate the distance between nodes for tranmission

End If;

End If;

Return;

End;

The above algorithm describes the cluster head selection, 
where the node and the initial energy are taken as the input. For all nodes, update the location and then split that nodes based on the location. If the initial energy of the node (NIE) is lesser than the total number of energy (NE), then it checks for the current node distance. If current node distance (NID) is less than the base station's distance (BD), then the cluster head will chose and allocate the slot accordingly. The distance of the node from the base station to destination is calculated to discover the route. The distance between the source node and all other nodes can be calculated by using the formula.

$\mathrm{D}=\sqrt{\left(y_{2}-y_{1}\right)^{2}+\left(x_{2}-x_{1}\right)^{2}}$

where, $(\mathrm{x} 1, \mathrm{y} 1)$ are the positions of the source node and (x2, y2) are the positions of the node from which the distance is calculated. After finding the shortest distance from the base station, the residual energy is calculated by using the crosslayer protocol.

Initially, the position of the node is updated and the neighbor node with one-hop distance is estimated. Furthermore, it verifies whether the node is in the neighbor list or not. If this case is true, then it checks whether the remaining energy is greater than the threshold value. If the condition satisfied, then the relay node is selected. Once the node is equal to the destination, then the data will processed and stop the relay station. Let us consider Ei be the initial energy of a node, and the residual energy is $E_{r e}$ of a node at time $t$, can be calculated by using the formula:

$\mathrm{E}_{\mathrm{re}}=\mathrm{E}_{\mathrm{i}}-\mathrm{E}_{\mathrm{c}}(\mathrm{t})$

where, $E_{c}$ is consumed energy and is estimated as follows:

$\mathrm{E}_{\mathrm{c}}(\mathrm{t})=\mathrm{E}_{\mathrm{tx}}+\mathrm{E}_{\mathrm{rx}}+\mathrm{E}_{\mathrm{i}}+\mathrm{E}_{\mathrm{s}}$

Here, the $E_{t x}$ denotes the transmission energy, $E_{r x}$ is the reception energy, $E_{i}$ is the idle energy, and $E s$ is the sleeping energy. In case, if the distance is within the transmission range and the node energy is less than the threshold value, then update the neighbor list of the source node. A route error message is sent back to the previous process. Once again the distance calculation is performed. If the energy value is greater than the threshold value, the route will be maintained.

\section{PROBLEM DEFINITION}

From the above study I have studied the different problems that are as follows:

- There is data duplication and data redundancy problem.

- Another problem is the network life time problem due to the redundancy.

- During the transmission energy is lost, so there is energy consumption problem.

- There is NP-hard scheduling problem that I have seen in the literature survey.

- Another problem is the more bandwidth and less network life time problem.

\section{METHODOLOGY}

This section defining the way of implementation that is to done in this research work. The basic function of opportunist routing (OR) is its ability to overhear the transmitted packet and to coordinate among relaying nodes. In OR, a candidate set is a potential group of nodes that is selected as the nexthop forwarders. Hence, each node in OR can use different potential paths to send packets toward the destination. Any of the candidates of a node that have received the transmitted packet may forward it. The decision of choosing the next forwarder is made by coordination between candidates that have successfully received the transmitted packet[16].

In this work WCA is designed with the help of Network Simulator. Since we assume that all nodes are identical and produce data at the same rate, to balance load in the system we have to balance the number of nodes in a cluster and the communication energy required per cluster head[17].

The node degree of a node vi is deduced as the cardinality of the set $\mathrm{N}\left(\mathrm{v}_{\mathrm{i}}\right)$ :

$$
\operatorname{deg}\left(v_{i}\right)=\left|N\left(v_{i}\right)\right|
$$

Based on the previous equations, we set our stability factor for each node $\mathrm{v}_{\mathrm{i}}$ as:

$$
\operatorname{STF}\left(v_{i}\right)=\operatorname{virD}\left(v_{i}\right) / \operatorname{deg}\left(v_{i}\right)
$$

We propose to calculate the relative dissemination degree. This parameter reflects the relative deviation of the number of neighbors in a current setting from that ideal.

$$
\beta\left(v_{i}\right)=\frac{\left|\delta-\operatorname{deg}\left(v_{i}\right)\right|}{\operatorname{deg}\left(v_{i}\right)}
$$

It is known that more power is required to communicate to a larger distance. Therefore, we are motivated to evaluate the energy consumption[19]. For this purpose, for every node $v_{i}$, we compute the sum of the distances $\mathrm{D}\left(\mathrm{v}_{\mathrm{i}}\right)$, with its neighbours, as:

$D\left(v_{i}\right)=\sum_{j=1}^{n} \operatorname{dis}\left(v_{i}, v_{j}\right)$

The cluster head selection process is composed of the following steps:

1. Find the neighbors (degree) of each node using

2. For each node, calculate the stability factor using (1)

3. For each node, apply the ANT processing to calculate the relative dissemination degree using (2)

4. Evaluate the energy consumption using (3)

5. Calculate the remaining battery energy of each node (RBE(vi))

6. Calculate the combined weight $\mathrm{W}(\mathrm{vi})$ for each for each

Node vi:

\section{$\mathrm{W}_{\mathrm{vi}}=\mathrm{D}(\mathrm{Vi}) * 0.2+\beta(\mathrm{Vi}) * 0.5+\mathrm{STF}^{*} 0.1+\mathrm{Mv}^{*} 0.2$}

7. Select the node not situated on the border and having the minimum weight Wvi as a cluster head.

8. Delete node vi and all itsN(vi) from $\mathrm{G}$.

9. Repeat the 7th and 8th steps until $G$ is empty. Awaiting Factor $=1$.

\section{RESULTS}


This includes the different snapshots that are on research work. These are given below:

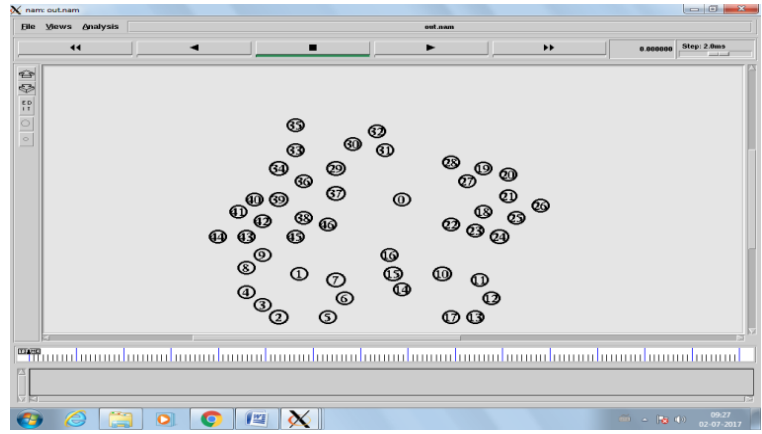

Figure 1: Input node distribution

Figure 1 is the input node distribution on the network. In this figure, different nodes are located at different positions on $\mathrm{X}$ axis and Y-axis. Each node has unique ID to identify the address .

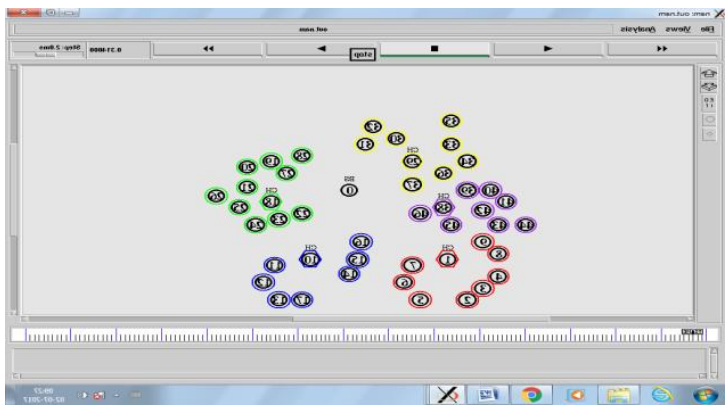

Figure 2: Node and Cluster distribution with color

In figure 2. is the processing of nodes with their color. Here Each node has different colors.Some node has red ,green, purple and yellow colors. Different color node has different clusters.

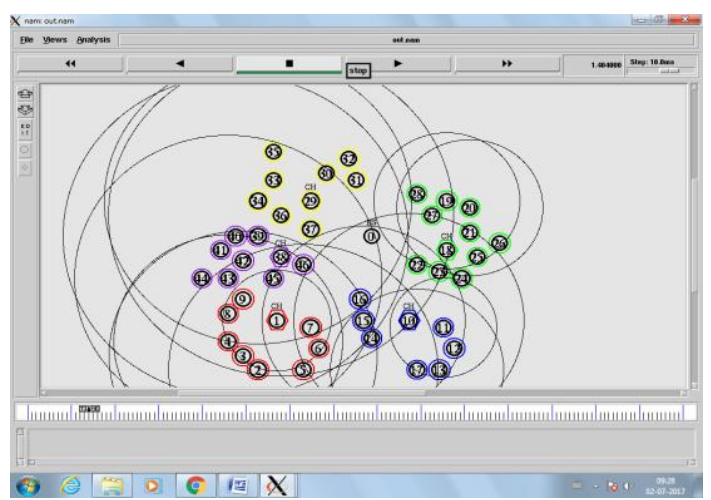

Figure 3: The transmission of signals in nodes and clusters

Figure 3. is the transmission of the signals in nodes and clusters. In this figure, different circles are displayed. It is the processing of signals between clusters and nodes..

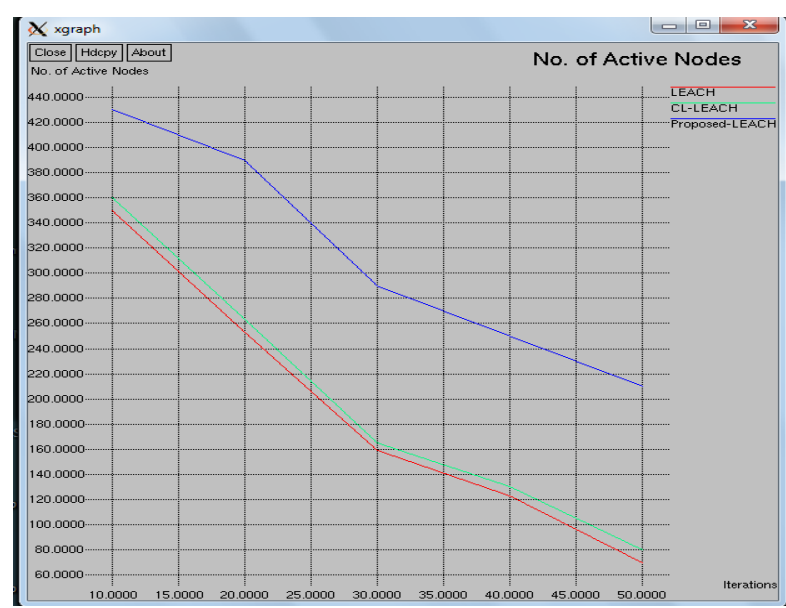

Figure 4: No. of Active Node w.r.t Iterations

Figure 4:is the No. of Active Node w.r.t Iterations of the network. Here red and Green line is the existing work and the Blue line is the proposed work of the data transmission.

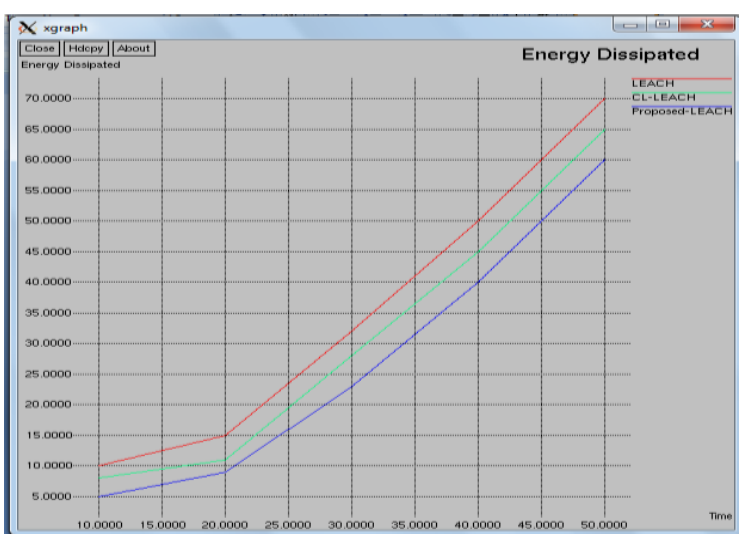

Figure 5: Energy_Dissipated w.r.t time

Figure 5: is the energy Dissipated of signals from node to node and node to the cluster head. It displays the total energy used in Network with time.

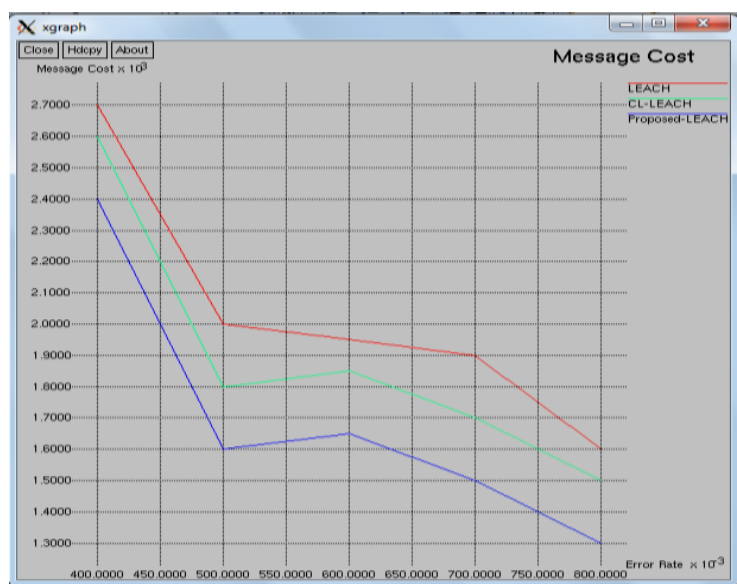

Figure 6: Message Cost w.r.t Error Rate

Figure 6: is Message Cost with Error rate during the transmission of data on network. It is the total cost of packet with respect to the error rate. 


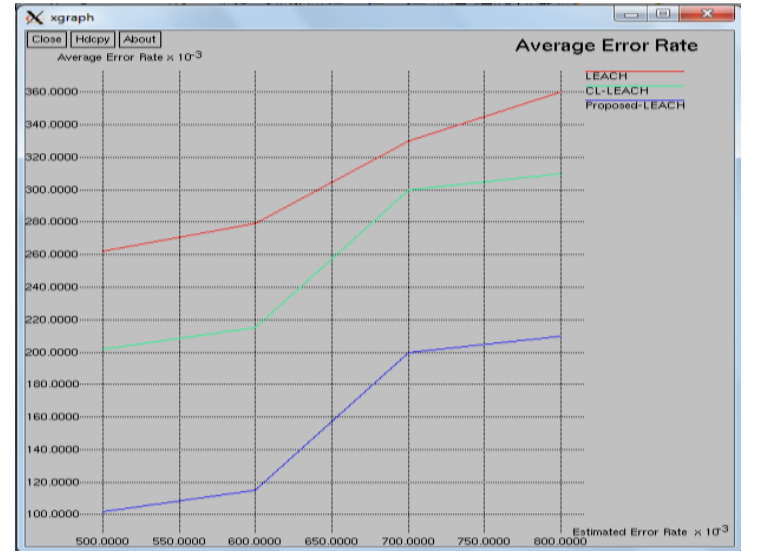

Figure 7: Average Error Rate w.r.t Estimated Error Rate

Figure 7 is the Average Error Rate w.r.t Estimated Error Rate. In this figure green line is the existing work and the Blue line is the proposed work.

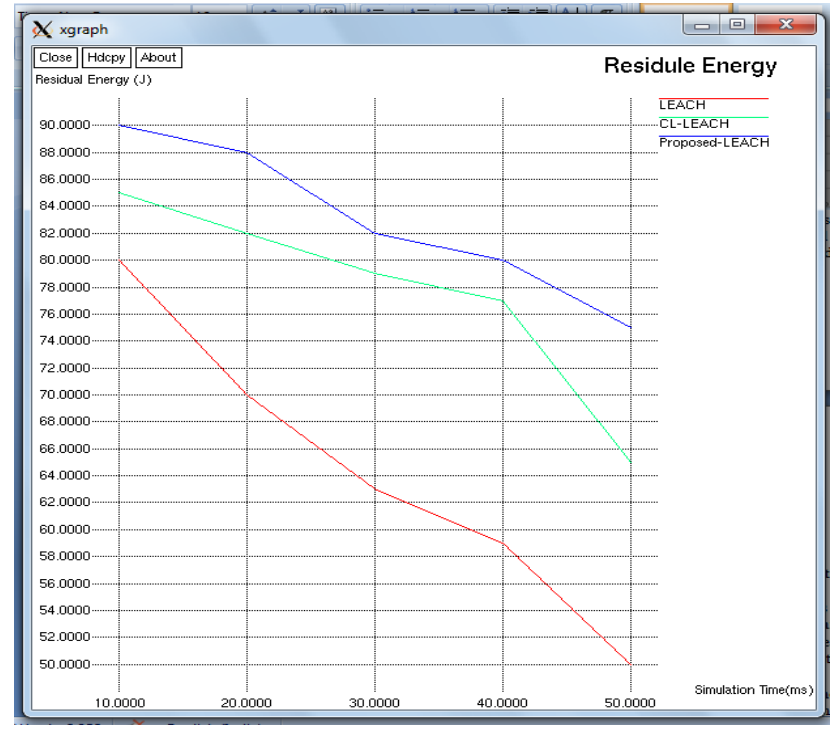

Figure 8: residual Energy w.r.t Simulation Time

\section{IX. CONCLUSION AND FUTURE SCOPE}

- Clustering is one of the important method to be applied in order to prolong the network lifetime of wireless sensor network. The existing protocols are not applicable to those WSNs that are deployed in large regions because it uses single hop routing where each sensor node can communicate directly to the cluster head and the base station. So, it causes problems of energy imbalance.

- The problem of unbalanced energy dissipation in the cluster based WSNs is investigated. In this, I even have designed wireless clump rule with the assistance node degree, Stability issue, and weight and cluster head choice.

- In this work, there is data duplication and data redundancy problem that I have faced and some other problem are the network life time problem due to the redundancy and transmission energy is lost, so there is energy consumption problem. Due to these problems some other problems like scheduling problem.

- All these Problems are resolved with the help of the hybrid techniques (combinations of Modified LEACH and $\mathrm{SCH}$ ) for data aggregations and for energy efficiency; the $\mathrm{CH}$ based routing algorithm on WSN is designed. The whole work is implemented in the NS2. The performance of proposed work is better than the existing work. The overall performance of work is $70 \%$.

\section{FUTURE WORK}

The research work includes a new energy-efficient routing algorithm for the software-defined wireless sensor networks. In our routing algorithm, the control nodes are assigned different tasks dynamically. It is further implemented with the help of other intelligent types like Honey Bee and ACO with PSO to get the real time work

\section{REFERENCES}

[1] Ablolfazl Afsharzadeh Kazerooni et.al. "Leach And Heed Clustering Algorithms In Wireless Sensor Networks: A Qualitative Study" Advances in Science and Technology Research Journal, Volume 9, Issue 25, 2015.

[2] Ablolfazl Afsharzadeh Kazerooni (2015) "Leach And Heed ClusteSring Algorithms In Wireless Sensor Networks: A Qualitative Study" ASTRJ, Volume 9, Issue 25,2015

[3] Annoa, J., Barollib, L., Durresic, A., Xhafad, F., \& Koyamae, A. (2008), "Performance evaluation of two fuzzy-based cluster head selection systems for wireless sensor networks", Mobile Information Systems, 4, 297 312.

[4] Baljinder Singh et.al.(2015)“Jamming attack in MANET: A Selected Review" International Journal of Advanced Research in Computer Science and Software Engineering , Volume 5, Issue 4, April 2015.

[5] Dawood.M.Sheik., et.al (2012), "Study of Energy Efficient Clustering Algorithm for Wireless Sensor Networks" International Journal of Emerging Research in Management \&Technology .

[6] Dehni. L., et.al (2006), "Power Control and Clustering in Wireless Sensor Networks," in Challenges in Ad Hoc Networking. vol. 197, K. Agha, et al., Eds., ed: Springer US.

[7] Grover.A., et.al (2014), "AOMDV with Multi-Tier Multi-Hop Clustering in Wireless Sensor Networks" Advanced Engineering Technology and Application,Adv. Eng. Tec. Appl. 3, No. 3, 29-33 .

[8] Harneet Kour et.al. "Hybrid Energy Efficient Distributed Protocol for Heterogeneous Wireless Sensor Network" International Journal of Computer Applications (0975 8887) Volume 4 - No.6, July 2010.

[9] Hashmi S and T. Moufth, (2003), "A New Transport Layer Sensor network protocol", IEEE Transactions, vol. 5,pp.118-156, Mar.2003

[10] Heinzelman.W.R., et.al (2000), “Energy-efficient communication protocol for wireless micro sensor networks," in System Sciences, 2000. Proceedings of the 33rd Annual Hawaii International Conference on.

[11] K. Saghar, D. Kendall, and A. Bouridane. Raeed: A solution for hello flood attack. In IEEE, International Bhurban Conference on Applied Sciences \& Technology Islamabad, (IBCAST 2015) Pakistan, 2015. 
[12] Khalid Hussain, Abdul Hanan Abdullah,(2013), "Cluster Head Election Schemes for WSN and MANETI: A Survey", ISSN 1818-4952 @ IDOSI Publications.

[13] Lynda Mokdad et.al.(2015) "DJAVAN: Detecting jamming attacks in Vehicle Ad hoc Networks " Elsevier B.V. All rights reserved 2015.

[14] M. Tariq and K. Saghar. Evaluation of a sensor network node communication using formal verification. In IEEE, International Bhurban Conference on Applied Sciences \& Technology Islamabad, (IBCAST 2015) Pakistan, 2015 .

[15] Maram Bani Younes et.al. (2015), "SCOOL: A Secure Traffic Congestion Control Protocol for VANETs" Wireless Communications and Networking Conference (WCNC): - Track 3: Mobile and Wireless Networks 2015 IEEE.

[16] Meng., et.al (2013), "An Energy Efficient Clustering
Scheme for Data Aggregation in Wireless Sensor Networks." Journal of Computer Science and Technology 28, no. 3 .

[17] OssamaYounis And Sonia Fahmy, Heed: -A Hybrid, Energy-Efficient, Distributed Clustering Approach For Ad-Hoc Sensor Networksll, (vol-3,issue- 4I 2009n 47907-2066, Usa).

[18] Rahmani.N., et.al (2010), "CAT: The New Clustering Algorithm Based on Two-Tier Network Topology for Energy Balancing in Wireless Sensor Networks," in Computational Intelligence and Communication Networks(CICN), 2010 International Conference on, 2010.

[19] Rohini Rawat et.al. (2015)"Mitigation of Jamming Attack in Vehicular Ad hoc Network" International Journal of Science, Engineering and Technology Research (IJSETR), Volume 4, Issue 6, June. 